\title{
Estudio del Impacto del Cambio Climático en los Caudales de Entrada al Embalse del Río Frío*
}

\section{Impact of Climate Change on the Input Flow of the Frio River Reservoir}

\author{
DOI: http://dx.doi.org/10.17981/ingecuc.12.1.2016.04 \\ Artículo de Investigación Científica - Fecha de recepción: 31 de agosto de 2015 - Fecha de aceptación: 9 de noviembre de 2015 \\ Mónica Tatiana Gómez Vargas \\ Magíster en Ingeniería Civil - Recursos Hidráulicos, Universidad Nacional de Colombia. Bogotá (Colombia). \\ mtgomezv@unal.edu.co
}

Para citar este artículo / To reference this paper:

M. T. Gómez Vargas "Estudio del impacto del cambio climático en los caudales de entrada al embalse del Río Frío", INGE CUC, vol. 12, no. 1, pp. 42-50, 2016. DOI: http://dx.doi.org/10.17981/ingecuc.12.1.2016.04

\begin{abstract}
Resumen-- Se evaluó cual podría ser la tendencia y la afectación en los caudales de entrada hasta el año 2100 del embalse que se tiene proyectado sobre el Río Frío para el abastecimiento de los municipios de Chía, Tenjo, Tabio, Cota y Cajicá considerando el RCP 4.5 y 8.5 , los cuales describen dos caminos del forzamiento radiativo hasta el año 2100. Estos tuvieron en cuenta las series asociadas a las simulaciones realizadas en los modelos climáticos globales CCSM4, IPSL CM5A -LR y MIROC ESM y los RCP. Se realizaron análisis comparativos para saber si las demandas proyectadas hasta el año 2070 podrán cumplirse al considerar el cambio climático. Se puso en evidencia que las demandas estimadas son elevadas y tienen un crecimiento exponencial, mientras que la oferta que se estimó tiene valores pequeños que no son suficientes para satisfacer los requerimientos de abastecimiento. Los caudales de entrada se esperan que tengan disminuciones de aproximadamente un $20 \%$.
\end{abstract}

Palabras claves--Cambio climático; variabilidad climática; escenario de cambio climático; gases efecto invernadero; modelo climático; downscaling; caudales de entrada.

\begin{abstract}
This paper poses an assessment of the trend and negative impact of the input flow for the projected reservoir in the Frio River that will cover the demand of the towns of Chía, Tenjo, Tabio, Cota, and Cajicá until the year 2100 considering RCP 4.5 and 8.5, which describe two ways of radiative forcing. Historical series and simulations based on global climate models like CCSM4, IPSL CM5A -LR, and MIROC ESM were used. Comparative analyses were performed to determine if the forecasted demands until the year 2070 can be supplied considering the climate change scenario. It is concluded that the estimated demands are elevated and have exponential growth, while the projected offer is not sufficient to satisfy supply requirements. The input flows are expected to decrease in an approximate of $20 \%$.
\end{abstract}

Keywords-- Climate Change; Climate Variability; Climate Change Scenario; Greenhouse Gases; Climate Model; Downscaling; Influx.

\footnotetext{
*Artículo de investigación científica derivado del proyecto de investigación titulado: "Estudio del impacto del cambio climático en las afluencias de un embalse ubicado en la jurisdicción de la CAR Cundinamarca, Colombia". Financiado por el autor. Fecha de inicio: diciembre 2013. Fecha de finalización: mayo 2015.
} 


\section{INTRODUCCIÓN}

El cambio climático es un tema que actualmente genera controversias de opiniones políticas, sociales, científicas y culturales. Algunos creen que las evidencias, como derretimiento parcial de capas polares y aumentos en el nivel del mar, son sólo suposiciones.

Para evaluar las posibles variaciones que se puedan relacionar con el cambio climático en el entorno, se han definido escenarios que buscan explicar las consecuencias potenciales de la influencia de las actividades humanas (crecimiento poblacional, desarrollo tecnológico, crecimiento económico, entre otros) sobre el clima, pues todas estas actividades son determinantes en la generación de gases efecto invernadero, los cuales son capaces de retener parte de la radiación que la superficie terrestre emite hacia el espacio.

Para analizar los escenarios de cambio climático se ha implementado el uso de modelos climáticos globales que buscan representar el comportamiento de variables como la precipitación y la temperatura de diversas zonas del mundo ante las condiciones que se encuentran implícitas en cada uno de los planteamientos de los escenarios. En algunos países han sido desarrollados modelos climáticos globales propios que buscan representar y simular el comportamiento de las variables climáticas a futuro. En Colombia no se cuenta con ningún grupo que realice estas investigaciones por lo que se deben adoptar los resultados de modelos generados en el mundo y realizar evaluaciones de la representación de los ciclos interanuales de precipitación y de temperatura en las zonas de nuestro país, así como de las condiciones de orografía dadas por ciertos modelos para que los resultados de los modelos puedan ser aplicados en el país.

El caso de aplicación es el embalse que se tiene proyectado sobre el río Frío, el cual actualmente se encuentra en etapa de prefactibilidad técnica, económica y ambiental y tiene como objetivo principal atender la demanda de los sistemas de acueducto de los municipios de Tabio, Tenjo, Cajicá, Chía y Cota.

Además, se busca verificar si las demandas estimadas hasta el año 2070 en los estudios que se han realizado previamente por la Corporación Autónoma Regional (CAR) podrán satisfacerse con las series de caudales de entrada resultantes al considerar el cambio climático.

\section{REVISIÓN LiterARIA}

Aunque no existe una definición única según el IPCC (Intergovernamental Panel of Climate Change), el cambio climático es una "importante variación estadística en el estado medio del clima o en su variabilidad que se puede deber a procesos naturales internos o a cambios del forzamiento externo o bien a cambios persistentes antropogénicos en la composición de la atmósfera o en el uso de las tierras" [1].

Colombia participa activamente en investigaciones para dar cumplimiento a los requerimientos de la Organización de las Naciones Unidas, que es la entidad que a nivel mundial busca proporcionar liderazgo y alentar la participación en el cuidado del medio ambiente inspirando, informando y facilitando a las naciones y los pueblos los medios para mejorar su calidad de vida sin comprometer la de las futuras generaciones.

Como parte de las investigaciones adelantadas actualmente se han definido cuatro escenarios denominados Caminos representativos de forzamiento radiativo, los cuales son una representación plausible de la evolución futura de las emisiones de sustancias que podrían ser radiativamente activas, por ejemplo, gases de efecto invernadero [2]. A su vez, el comportamiento de los caminos representativos se relaciona mediante los modelos climáticos globales, los cuales buscan representar con la menor incertidumbre el comportamiento de parámetros como temperatura y precipitación. Aunque existen muchos modelos climáticos globales para representar las condiciones climáticas y orográficas del país, el IDEAM ha realizado evaluaciones del desempeño de los mismos, encontrando que los modelos CCSM4, MIROC- ESM, IPSL - CM5A - LR [3] tienen un buen desempeño.

Es importante resaltar que los escenarios de cambio climático no son pronósticos ni predicciones, buscan relacionar la influencia del hombre y sus actividades con la variación del clima de una zona y sirven como herramienta de planificación en la toma de decisiones de diferentes proyectos, de definición de medidas de adaptación, de identificación de vulnerabilidad, entre otros aspectos.

Actualmente, en el mundo se están implementando análisis de cambio climático para validar si existirán cambios en la producción energética de los proyectos. Un ejemplo es el caso del estudio realizado en Chile donde se evaluó si el cambio climático podrá impactar en la generación de las centrales localizadas en la cuenca del Alto Cachapoal; la conclusión muestra cómo la variación simulada en los caudales afluentes a los proyectos está relacionada con la disminución en la potencia generada en el periodo analizado [4]. En California se realizaron estudios para evaluar la implicaciones de los cambios en la temperatura y la precipitación asociados al cambio climático en la importación de energía a la zona, encontrando que aumentarán estas importaciones en un 35\%, considerando que se esperan aumentos en la temperatura de $4^{\circ}$ media y una disminución del $25 \%$ en la precipitación promedio [5]. 


\section{III.Metodología}

Con el fin de estimar la variación en los caudales de entrada al embalse del río Frío hasta el año 2100 considerando los escenarios de cambio climático vigentes se describe a continuación de manera breve la metodología desarrollada:

Se realizó la delimitación de la cuenca hidrográfica de la corriente donde se localizará el proyecto, así mismo se identificaron y recopiló la información de las estaciones representativas de la zona que permitieran conocer el comportamiento de variables como la precipitación, temperatura y caudales durante la historia de operación de la estación.

Cuando se realizan análisis de cambio climático deben definirse dos periodos, uno que se denomina "periodo presente", el cual está en función del periodo de registro reportado por la estación hidrométrica representativa y el límite superior será el año 2005, este periodo será base para todas las comparaciones que se realicen; y otro periodo denominado "futuro" el cual estará comprendido entre $(2006$ - 2100) y será bajo el cual se estimarán las tendencias y comportamientos de las afluencias.

Una vez se tienen definidos los periodos presente y futuro, se descargaron del portal de The Earth System Grid Federation (ESGF) los valores de las variables de precipitación y temperatura para los periodos mencionados. Estos datos son el resultado de combinar las simulaciones resultantes de los modelos climáticos globales seleccionados, los RCP o caminos representativos y la resolución a la que se vayan a realizar los análisis, principalmente.

Es difícil comparar los resultados obtenidos entre un modelo climático global y los valores de las variables registradas por las estaciones localizadas en la cuenca de estudio pues usualmente las grillas donde vienen presentados estos modelos tienen un tamaño que se encuentra en un rango de aproximadamente $100 * 100 \mathrm{~km}^{2}$ a $282 * 282$ $\mathrm{km}^{2}$ y se hace necesario realizar comparaciones en cuencas que tienen un tamaño menor. Por esto se han desarrollado técnicas que permitan solucionar esta diferencia de resolución, es decir, hacer comparables los resultados generados por los modelos climáticos globales con los registros presentados por las estaciones. Uno de estos métodos es el factor delta [6], el cual relaciona los valores medios mensuales multianuales de las series de los datos registrados por la estación y de los datos simulados por los modelos climáticos globales seleccionados. A continuación en (1) se presenta la ecuación que describe dicho método:

$$
X_{i, j}^{*}=\propto_{j} X_{i j}^{\bmod }
$$

Donde:
$X_{i, j}^{*}$ : Es el valor ajustado de la variable X (precipitación del mes j del año i).

$X_{i j}^{\text {mod }}$ : Es el valor modelado de la variable para el mes j y año i (periodo futuro).

Así mismo, en (2)

$$
\propto_{j}=\frac{X_{i, j}^{o b s}}{X_{i j}^{m o d}}
$$

Donde:

$X_{\mathrm{i}, \mathrm{j}}^{\text {obs }}$ : Es el valor medio mensual multianual de la precipitación del mes j registrada por la estación.

$X_{i, j}^{\text {mod }}$ : Es el valor medio mensual multianual de la precipitación del mes j simulada.

Nota: Se estima un factor para cada mes.

Una vez se cuenta con las series ajustadas hasta el año 2100, se prosigue con la aplicación del protocolo de modelación. El modelo hidrológico que se seleccionó corresponde al modelo paramétrico a, b, c y d de Thomas, el cual es un modelo hidrológico simple para simular caudales en respuesta a la precipitación y la evapotranspiración potencial. Fue desarrollado por Harold A. Thomas Jr. en 1981 [7]. Este modelo puede aplicarse de dos maneras, el primero es la manera inversa, mediante el cual se pueden estimar los valores de los parámetros si se conocen los datos de entrada de precipitación y evapotranspiración, adicionalmente se aplica en la etapa de calibración del modelo. Posteriormente, se aplica el método de manera directa, el cual conocidos los parámetros del modelo y los datos de precipitación y evapotranspiración para el periodo futuro permite estimar los caudales de entrada hasta el año 2100.

A las series de precipitación, temperatura y caudales obtenidas hasta el año 2100 se le realizaron pruebas de tendencia para analizar cuál es el comportamiento que se espera.

Una vez se estimaron los valores de los caudales de entrada al embalse hasta el año 2100 considerando el cambio climático, se realizó un análisis de tendencia gráfico para conocer el comportamiento que se espera de la serie hasta este periodo. Por último, se estimaron los índices de escasez y presión hídrica del proyecto hasta el año 2070, y se compararon con las demandas que habían sido estimadas por la CAR hasta este año.

\section{Resultados}

Se delimitó la cuenca hidrográfica del proyecto y se realizó la identificación y recopilación de registros de las estaciones representativas (Fig. 1). Se recopilaron los registros de precipitación en el periodo 1988 - 2013, de temperatura en el periodo 1991 - 2002 y, en el caso de la estación liminimétrica Puente La Virginia, los registros recopilados corresponden al periodo 1947- 2014. 


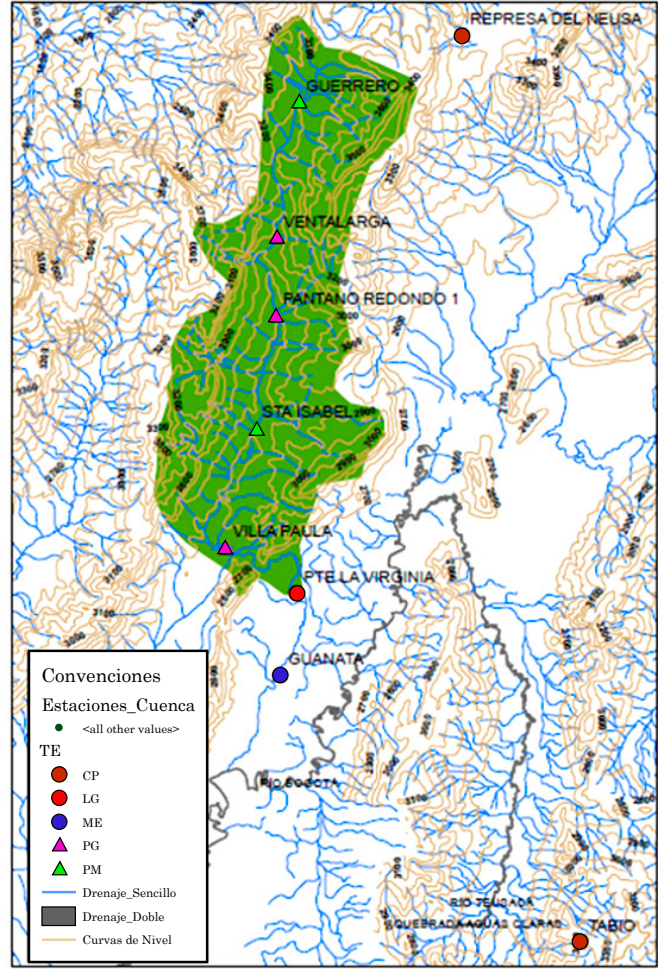

Fig. 1. Localización de estaciones representativas. Fuente: [8]

Se realizaron análisis de tendencia para los registros recopilados de las estaciones pluviométricas, climatológicas y para la estación limnigráfica Puente La Virginia que fueron seleccionadas como representativas en la zona, observándose en las estaciones pluviométricas una tendencia de aumento en los registros presentados en el periodo 1998 - 2013. Los registros de las estaciones que registraban temperatura no presentan una tendencia definida; y finalmente, la estación limnigráfica Puente La Virginia presenta una tendencia a la disminución. A continuación, en las Figs. 2, 3 y 4 se presentan algunas de las tendencias identificadas para las series de los diferentes parámetros.

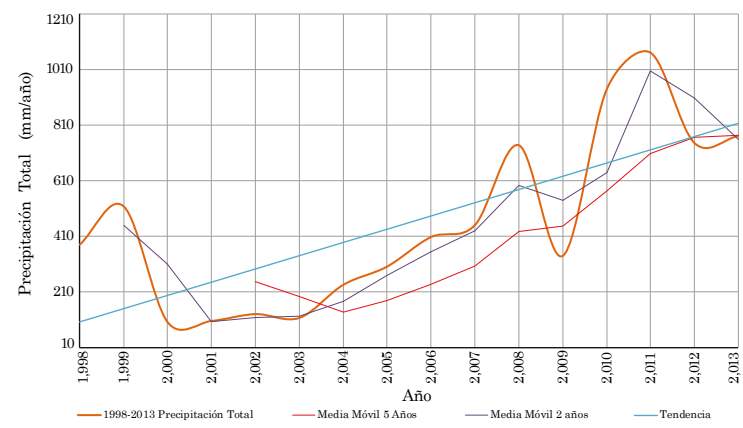

Fig. 2. Análisis de tendencia de la serie de precipitación de la estación Santa Isabel (Periodo 1998 - 2013). Fuente: [8]

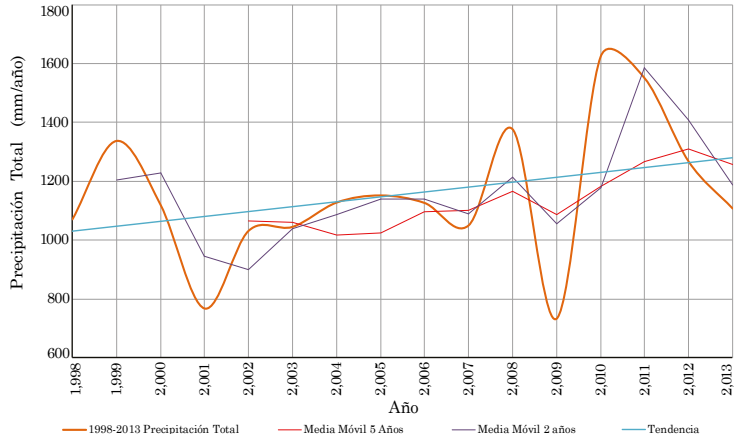

Fig. 3. Análisis de tendencia de la serie de precipitación de la estación Ventalarga (Periodo 1998 - 2013). Fuente: [8]

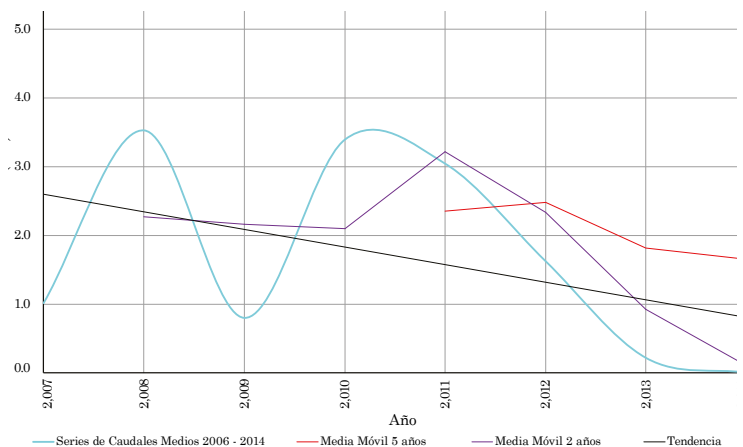

Fig. 4. Serie de caudales de la estación limnigráfica Puente La Virginia (Periodo 1947 - 2005).

Fuente: [8]

La extracción de datos para los periodos definidos como actual y futuro se realizó bajo los siguientes parámetros:

- Proyecto (el proyecto evaluado es el CMIP5).

- Modelo (se escoge cuál modelo climático global a analizar entre CCSM4, MIROC-ESM, IPSLCM5A-LR, estos modelos fueron seleccionados por tener el mejor desempeño en evaluaciones realizadas por el IDEAM [3]).

- Nombre de la variable (variables descargadas en el presente informe: precipitación y temperatura).

- Experimento (corresponde a cada RCP, los RCP analizados en el presente estudio son RCP $4.5 \mathrm{y}$ RCP 8.5, ya que el RCP 2.5 es un escenario optimista que no ha presentado el comportamiento del forzamiento radiativo que se tenía proyectado en lo que va corrido del siglo, y por otra parte, para el RCP 6.0 no todos los modelos climáticos globales que fueron base para la definición de los RCP vigentes realizaron simulaciones para todas las variables climáticas que se necesitan para realizar los análisis descritos).

- Frecuencia (especifica la frecuencia de los datos a la que se van a realizar los análisis: diaria y mensual)

- Ensamble (r1ip1). 
Teniendo en cuenta que los datos simulados presentan una resolución que no es comparable con los datos reportados por las estaciones de la zona de estudio, se realiza la estimación de factores delta, los cuales relacionan los caudales medios mensuales de los caudales simulados y registrados por la estación base para el periodo de calibración del modelo hidrológico que será seleccionado y disminuye la resolución de los registros generados por los modelos para hacerlos comparables con los registros de las estaciones localizadas en la zona de estudio. A continuación en la Fig. 5 se presenta la localización de la cuenca del proyecto delimitada respecto a las grillas resultantes del modelo CCSM4.

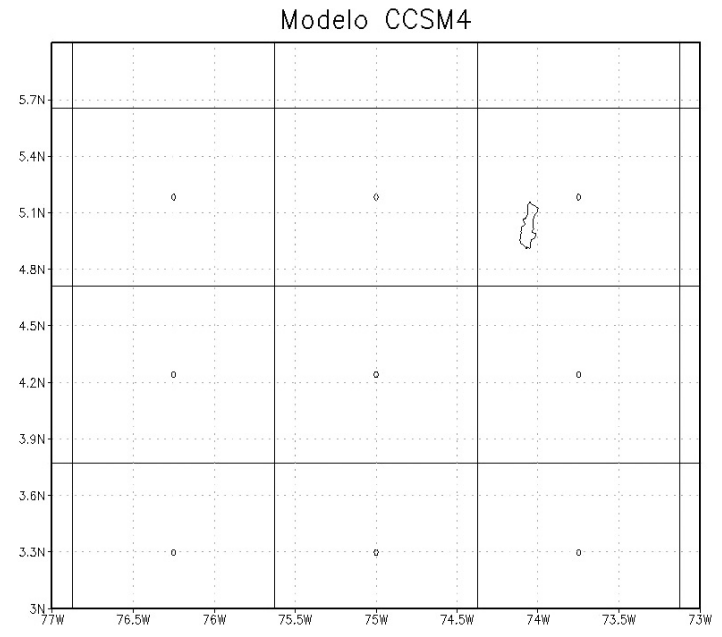

Fig. 5. Localización de la cuenca de estudio respecto a la grilla del modelo CCSM4. Fuente: [8]
Fueron estimadas las series futuras de precipitación y temperaturas ajustadas mediante los factores delta para cada mes en cada modelo. Los resultados de los factores delta en promedio para los doce meses del año se presentan a en la Tabla I.

La calibración del modelo de Thomas inverso se realizó mediante una calibración automática que consideró como función objetivo el logaritmo natural del error cuadrático medio. El resultado obtenido fueron los valores de los parámetros que representan las características físicas del área en estudio; adicionalmente, estos parámetros fueron los que mejor representaban las series de caudales registradas por la estación liminimétrica Puente La Virginia. Estos valores habían sido definidos en un estudio previo realizado por la CAR [9] y fueron tomados como referencia, aunque consideraron un periodo de calibración y métodos de evapotranspiración diferentes, así mismo, tenía en cuenta los escenarios de cambio climático que ya no están vigentes. En la Tabla II se presentan los valores de los parámetros mencionados.

Posteriormente se realizó la aplicación del modelo de Thomas directo con el fin de estimar las series de caudales de entrada hasta el periodo 2100 suponiendo que los parámetros a, b, c y d estimados mediante la aplicación del modelo de Thomas inverso serán invariables en el tiempo, esto al tener en cuenta que los parámetros están asociados con características de la cuenca como la permeabilidad, el uso del suelo y el flujo base, de los cuales no se tiene identificado cómo sería la variación en el tiempo al considerar los efectos

Tabla I. Promedios Mensuales de Factores Delta

\begin{tabular}{|c|c|c|c|c|c|}
\hline Código & Nombre & Periodo de Aplicaciòn & CCMS4 & MIROC ESM & IPSL CM5A -LR \\
\hline 2120026 & VENTALARGA & $1988-2005$ & 0.95 & 0.51 & 0.55 \\
\hline 2120176 & STA ISABEL & $1988-2005$ & 0.24 & 0.16 & 0.19 \\
\hline 2120188 & VILLA PAULA & $1992-2005$ & 0.65 & 0.48 & 0.55 \\
\hline 2120214 & GUERRERO & $1988-2005$ & 0.95 & 1.91 & 1.20 \\
\hline 2120106 & PANTANO REDONDO 1 & $1988-2005$ & 1.02 & 0.56 & 0.59 \\
\hline
\end{tabular}

Fuente: [8]

Tabla II. Valores de los Parámetros A, B, C, D obtenidos y de Referencia

\begin{tabular}{|c|c|c|c|c|c|c|c|}
\hline $\begin{array}{l}\text { Nombre dado al grupo de } \\
\text { parámetros }\end{array}$ & $\begin{array}{l}\text { Método de } \\
\text { Evapotranspiración }\end{array}$ & a & b & c & d & Swo & Sgo \\
\hline$a, b, c, d$ & ETP promedio & 0,98 & 145 & 0,01 & 0,89 & 72,01 & 0,12 \\
\hline Valores de referencia estudio-CAR & & 0,77 & 180,55 & 0,07 & 0,22 & & \\
\hline
\end{tabular}

Fuente: [8] 
de cambio climático. Como resultado pudo observarse que mediante las condiciones dadas por el modelo, el RCP 8.5 seguirá manteniendo la tendencia a disminución de los caudales de entrada en la estación de caudales representativa de la zona del proyecto. En la Fig. 6 se presenta la serie de caudales afluentes hasta el año 2100 y la tendencia de disminución de caudales que se proyecta.

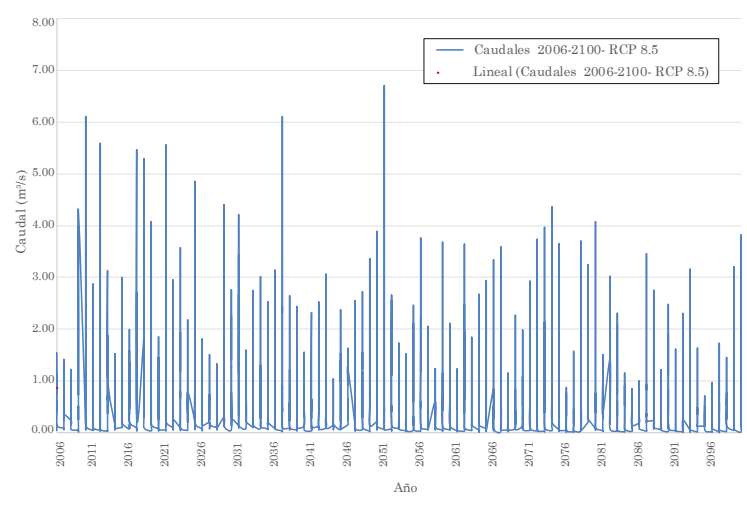

Fig. 6 Series de Caudales Simulados 2006 - 2100. Fuente: [8]

Por último se realizó la comparación de las demandas para el proyecto que fueron estimadas por la CAR [10] hasta el año 2070 y se compararon con las series de ofertas estimadas hasta ese mismo periodo. La demanda presentada por la CAR tiene en cuenta el abastecimiento de cada uno de los municipios (sectores urbano y rural) y se determinó el caudal máximo demandado desde la fuente (QMDFUENTE) para cada vigencia a partir de la estimación de las necesidades reales de captación así: QMDFUENTE: caudal máximo diario, (QMD) + las pérdidas de agua cruda en la aducción y conducción $(5 \%)+$ pérdidas de agua cruda en la planta de tratamiento (3\%), para finalmente determinar el caudal máximo demandado total. Mientras que la oferta neta se determinó tomando las series de caudales estimadas bajo los escenarios de cambio climático y considerando la diferencia entre el caudal que es excedido el $90 \%$ del tiempo y el caudal ecológico, el cual fue definido en el informe realizado por la CAR como el $25 \%$ del caudal medio mensual multianual más bajo de la corriente en estudio.

Tomando como referencia las series de demandas y ofertas hasta el año 2070, se estimó el índice de escasez para evaluar la relación existente entre la oferta hídrica disponible y las condiciones de demanda predominantes en la zona. "El índice de escasez es la relación porcentual de la demanda de agua ejercida por las actividades sociales y económicas en su conjunto para su uso y aprovechamiento con la oferta hídrica disponible (neta)" [11].

El índice de escasez se agrupa en cinco categorías, las cuales se presentan en la Tabla III:
Tabla III. Clasificación del Índice de Escasez

\begin{tabular}{|c|c|c|}
\hline Categoría & $\begin{array}{c}\text { Índice de } \\
\text { Escasez }\end{array}$ & Características \\
\hline No significativo & $<1 \%$ & $\begin{array}{c}\text { Demanda no significativa } \\
\text { con relación a la oferta. }\end{array}$ \\
\hline Mínimo & $1-10 \%$ & $\begin{array}{c}\text { Demanda muy baja con } \\
\text { respecto a la oferta. }\end{array}$ \\
\hline Medio & $11-20 \%$ & $\begin{array}{c}\text { Demanda baja con respecto } \\
\text { a la oferta. }\end{array}$ \\
\hline Medio alto & $21-50 \%$ & Demanda apreciable. \\
\hline Alto & $>50 \%$ & $\begin{array}{c}\text { Demanda alta con respecto } \\
\text { a la oferta. }\end{array}$ \\
\hline
\end{tabular}

Fuente: [11]

En la Tabla IV se presentan los resultados obtenidos para el escenario RCP 8.5 con modelo MIROC ESM. Sólo se presenta esta condición a manera de ejemplo, pues los resultados obtenidos bajo los demás escenarios y modelos presentan resultados similares.

Al realizar esta comparación se observó (Fig. 7) que el recurso hídrico en la zona tiene una gran presión y el valor de los índices de presión y de escasez presentan valores fuera de los límites establecidos por la autoridad Nacional IDEAM en el Estudio Nacional del Agua [10]. Puede observarse que la oferta hídrica presenta pequeños valores, respecto a los valores solicitados según lo establecido en el informe elaborado por la CAR [11].

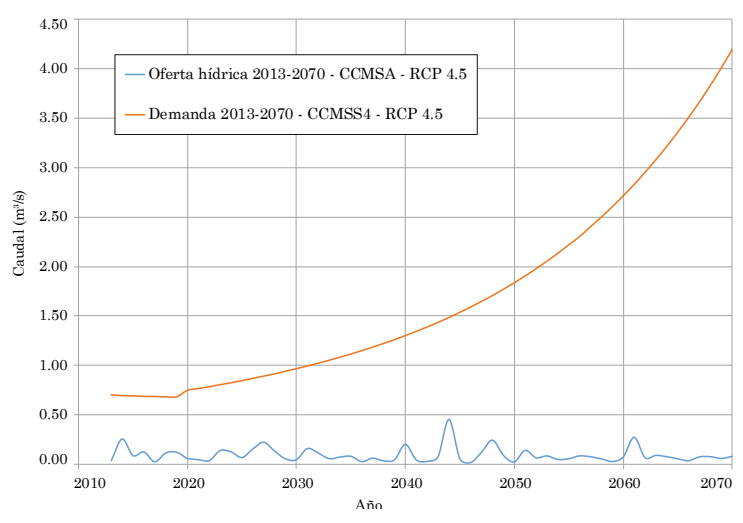

Fig. 7. Oferta hídrica vs demanda 2013 -2070. Fuente: [8]

\section{Conclusiones}

- Se realizaron análisis de tendencia para los registros recopilados de las estaciones pluviométricas, climatológicas y para la estación limnigráfica Puente La Virginia que fueron seleccionadas como representativas en la zona, observándose en las estaciones pluviométricas una tendencia de aumento en los registros presentados en el periodo 1998 - 2013. Los registros de las estaciones 
TABLA IV.

\begin{tabular}{|c|c|c|c|c|c|c|c|}
\hline Nombre dado al grupo de parámetros & Método de evapotranspiración & a & b & c & d & Swo & Sgo \\
\hline CAR 3 & ETP Promedio & 0.98 & 145,00 & 0,01 & 0,89 & 72,01 & 0,12 \\
\hline
\end{tabular}

Fuente: [8]

que registraban temperatura no presentan una tendencia definida, y finalmente, la estación limnigráfica Puente La Virginia presenta una tendencia a la disminución.

- El grupo de parámetros que relaciona las características físicas de la cuenca como la permeabilidad y capacidad de almacenamiento, representa las series de caudales registradas por la estación representativa Puente La Virginia y origina que las series simuladas tengan características estadísticas similares a la serie de la estación base, fueron los denominados CAR 3. Los valores seleccionados se presentan a continuación en la tabla IV.

La simulación realizada tuvo en cuenta el logaritmo natural del error cuadrático medio Ln (RSME).

- La tendencia que se espera para la serie de afluencias al embalse en el periodo $2006-2100$ del río Frío es de disminución, tal como se pone en evidencia en la serie de la estación Puente La Virginia en sus registros desde 1947 hasta el año 2013, año hasta el que fue posible recopilar información. El valor promedio se espera que sea del $20 \%$ y es resultante de los valores que presentan los modelos CCSM4 y el IPSL CM5A - LR.

- Se realizó la estimación de los índices de escasez y de presión hídrica a nivel anual con el fin de verificar si las demandas proyectadas para abastecer los sistemas de acueducto de los municipios de Tabio, Tenjo, Cajicá, Chía y Cota pueden ser atendidas con los caudales de entrada generadas desde 2013 hasta 2070 al considerar el cambio climático. De lo anterior pudo concluirse que las demandas presentan una forma de crecimiento exponencial mientras que las ofertas varían en rangos pequeños que no son suficientes para atender las demandas estimadas hasta el año 2070, generando valores del índice de escasez y de presión hídrica fuera de los limites definidos por el IDEAM en el Estudio Nacional del Agua. Lo anterior sugiere una revisión de las demandas solicitadas y la búsqueda de fuentes adicionales de abastecimiento para cumplir con las necesidades definidas.

\section{REFERENCIAS}

[1] Working Group I, Contribution to the Fifth As sessment Report of the Intergovernmental Panel on Climate Change, Climate Change 2013: The Physical Science Basis. New York: Cambridge University Press, 2013.

[2] Contribución de los Grupos de trabajo I, II y III al Cuarto Informe de evaluación del Grupo Intergubernamental de Expertos sobre el Cambio Climático, Cambio climático 2007, Informe de síntesis. Ginebra: Grupo Intergubernamental de Expertos sobre el Cambio Climático, 2007.

[3] Instituto de Hidrología, Meteorología y Estudios Ambientales, "Nuevos Escenarios de Cambio Climático, Nivel Nacional - Departamental,” Bogotá, 2015.

[4] T. I. Gómez Zavala, "Evaluación del impacto del cambio climático en centrales hidroeléctricas de la cuenca del Alto Cachapoal,” M.S. thesis, Dept. Sci. Phys aand Math., Universidad de Chile, Chile, 2013.

[5] L. L. Dale, N. Karali, D. Millstein, M. Carnall, S. Vicuña, N. Borchers, E. Bustos, J. O'Hagan, D. Purkey, C. Heaps, J. Sieber, W. D. Collins, and M. D. Sohn, "An integrated assessment of water-energy and climate change in sacramento, california: how strong is the nexus?," Clim. Change, vol. 132, no. 2, pp. 223235, Jul. 2015. DOI: 10.1007/s10584-015-1370-x

[6] J. D. Pabón Caicedo, El cambio climático en el territorio de la Corporación Autónoma Regional de Cundinamarca. CAR, 2011.

[7] H. A. Thomas, "Improved methods for national water assessment, water resources contract: WR15249270," 1981.

[8] M. T. Gómez Vargas, "Estudio del impacto del cambio climático en las afluencias de un embalse ubicado en la jurisdicción de la CAR Cundinamarca, Colombia”, Universidad Nacional de Colombia, 2015.

[9] Corporación Autónoma Regional, "Modelación hidrológica de los impactos de los escenarios de ec en la escorrentía superficial y en un caso de estudio de un acuífero de la jurisdicción CAR en las cuencas hidrográficas, que sirva de base para avanzar en las Evaluaciones del ERA,” Bogotá, 2014.

[10] Corporación Autónoma Regional, "Estudios de prefactibilidad técnica, económica y ambiental del proyecto de embalse en la cuenca del río frío convenio interadministrativo de asociación no 529 de 2012- Capítulo Planeamiento y Disponibilidad de Agua," Bogotá, 2014.

[11] Instituto de Hidrología, Meteorología y Estudios Ambientales, "Estudio Nacional del Agua," 2010. 
TABLA V. ÍNDICE DE ESCASEZ ESTIMADO BAJO EL ESCENARIO MIROC ESM- RCP 8.5

\begin{tabular}{|c|c|c|c|c|c|}
\hline AÑ̃ & Q90 & Caudal ecológico $\left(\mathrm{m}^{3} / \mathrm{s}\right)$ & Caudal de reparto $\left(\mathrm{m}^{3} / \mathrm{s}\right)$ & Demanda $\left(\mathrm{m}^{3} / \mathrm{s}\right)$ & Índice de escasez \\
\hline 2013 & 0,143 & 0,024 & 0,12 & 0,70 & 592,12 \\
\hline 2014 & 0,149 & 0,031 & 0,12 & 0,70 & 591,14 \\
\hline 2015 & 0,161 & 0,035 & 0,13 & 0,69 & 549,23 \\
\hline 2016 & 0,149 & 0,024 & 0,12 & 0,69 & 552,87 \\
\hline 2017 & 0,110 & 0,025 & 0,08 & 0,69 & 809,76 \\
\hline 2018 & 0,078 & 0,018 & 0,06 & 0,68 & 1134,62 \\
\hline 2019 & 0,114 & 0,027 & 0,09 & 0,68 & 785,60 \\
\hline 2020 & 0,141 & 0,031 & 0,11 & 0,75 & 680,54 \\
\hline 2021 & 0,082 & 0,013 & 0,07 & 0,77 & 1110,95 \\
\hline 2022 & 0,132 & 0,026 & 0,11 & 0,79 & 743,83 \\
\hline 2023 & 0,122 & 0,025 & 0,10 & 0,81 & 837,06 \\
\hline 2024 & 0,082 & 0,014 & 0,07 & 0,83 & 1209,94 \\
\hline 2025 & 0,029 & 0,004 & 0,02 & 0,85 & 3400,72 \\
\hline 2026 & 0,098 & 0,018 & 0,08 & 0,87 & 1090,23 \\
\hline 2027 & 0,048 & 0,004 & 0,04 & 0,89 & 2035,60 \\
\hline 2028 & 0,089 & 0,022 & 0,07 & 0,92 & 1373,59 \\
\hline 2029 & 0,099 & 0,023 & 0,08 & 0,94 & 1245,94 \\
\hline 2030 & 0,097 & 0,023 & 0,07 & 0,97 & 1310,22 \\
\hline 2031 & 0,075 & 0,013 & 0,06 & 1,00 & 1598,23 \\
\hline 2032 & 0,094 & 0,017 & 0,08 & 1,02 & 1325,22 \\
\hline 2033 & 0,075 & 0,017 & 0,06 & 1,05 & 1805,08 \\
\hline 2034 & 0,074 & 0,017 & 0,06 & 1,08 & 1887,93 \\
\hline 2035 & 0,093 & 0,022 & 0,07 & 1,12 & 1571,02 \\
\hline 2036 & 0,109 & 0,024 & 0,08 & 1,15 & 1360,02 \\
\hline 2037 & 0,094 & 0,021 & 0,07 & 1,19 & 1637,54 \\
\hline 2038 & 0,112 & 0,022 & 0,09 & 1,22 & 1359,57 \\
\hline 2039 & 0,111 & 0,023 & 0,09 & 1,26 & 1433,56 \\
\hline 2040 & 0,080 & 0,015 & 0,06 & 1,30 & 2003,44 \\
\hline 2041 & 0,145 & 0,034 & 0,11 & 1,35 & 1209,07 \\
\hline 2042 & 0,112 & 0,025 & 0,09 & 1,39 & 1596,49 \\
\hline 2043 & 0,111 & 0,022 & 0,09 & 1,44 & 1618,76 \\
\hline 2044 & 0,095 & 0,019 & 0,08 & 1,49 & 1956,51 \\
\hline 2045 & 0,108 & 0,024 & 0,08 & 1,54 & 1823,18 \\
\hline 2046 & 0,085 & 0,021 & 0,06 & 1,59 & 2486,89 \\
\hline 2047 & 0,094 & 0,022 & 0,07 & 1,65 & 2289,63 \\
\hline
\end{tabular}


Sig...

\begin{tabular}{|c|c|c|c|c|c|}
\hline AÑo & Q90 & Caudal ecológico $\left(\mathrm{m}^{3} / \mathrm{s}\right)$ & Caudal de reparto $\left(\mathrm{m}^{3} / \mathrm{s}\right)$ & Demanda $\left(\mathrm{m}^{3} / \mathrm{s}\right)$ & Índice de escasez \\
\hline 2048 & 0,127 & 0,025 & 0,10 & 1,71 & 1679,43 \\
\hline 2049 & 0,081 & 0,017 & 0,06 & 1,77 & 2787,26 \\
\hline 2050 & 0,107 & 0,024 & 0,08 & 1,84 & 2220,38 \\
\hline 2051 & 0,111 & 0,020 & 0,09 & 1,91 & 2106,05 \\
\hline 2052 & 0,099 & 0,019 & 0,08 & 1,98 & 2470,09 \\
\hline 2053 & 0,144 & 0,018 & 0,13 & 2,05 & 1636,12 \\
\hline 2054 & 0,106 & 0,018 & 0,09 & 2,13 & 2427,45 \\
\hline 2055 & 0,109 & 0,020 & 0,09 & 2,22 & 2484,25 \\
\hline 2056 & 0,095 & 0,018 & 0,08 & 2,30 & 2972,79 \\
\hline 2057 & 0,076 & 0,018 & 0,06 & 2,40 & 4173,50 \\
\hline 2058 & 0,085 & 0,016 & 0,07 & 2,50 & 3623,00 \\
\hline 2059 & 0,089 & 0,021 & 0,07 & 2,60 & 3835,18 \\
\hline 2060 & 0,095 & 0,021 & 0,07 & 2,71 & 3673,75 \\
\hline 2061 & 0,051 & 0,011 & 0,04 & 2,83 & 7070,22 \\
\hline 2062 & 0,080 & 0,016 & 0,06 & 2,95 & 4628,91 \\
\hline 2063 & 0,136 & 0,030 & 0,11 & 3,08 & 2911,11 \\
\hline 2064 & 0,080 & 0,019 & 0,06 & 3,21 & 5198,45 \\
\hline 2065 & 0,080 & 0,013 & 0,07 & 3,36 & 5016,36 \\
\hline 2066 & 0,100 & 0,015 & 0,08 & 3,51 & 4126,05 \\
\hline 2067 & 0,097 & 0,023 & 0,07 & 3,66 & 4982,80 \\
\hline 2068 & 0,080 & 0,016 & 0,06 & 3,83 & 5969,15 \\
\hline 2069 & 0,054 & 0,012 & 0,04 & 4,01 & 9478,36 \\
\hline 2070 & 0,091 & 0,019 & 0,07 & 4,19 & 5801,28 \\
\hline
\end{tabular}

Fuente: [8] 